\title{
ALIMENTATION ARTIFICIELLE DE LA NAPPE DES ALLUVIONS DE LA BASSE-DURANCE
}

\author{
PAR \\ R. MULLER-FEUGA * \\ ET \\ P. RUBY * *
}

La Durance, dernier aflluent du Rhône, est en cours d'équipement par Electricité de France. Dans la partie inférieure de la rivière, l'aménagement de la Basse-Durance se développe dans une région de plaines alluviales où l'agriculture est florissante grâce à l'arrosage de 75000 ha : le débit maximal dérivé pour l'irrigation atteint en été $114 \mathrm{~m}^{3} / \mathrm{s}$.

L'influence de la dérivation des eaux de la rivière dans un canal industriel, d'abord parallèle à la Durance, puis quittant la vallée de celle-ci pour aboutir à l'étang de Berre (par une cascade de 5 usines d'une productibilité annuelle de $2300 \mathrm{GWh}$ ) a longtemps préoccupé les représentants des riverains qui craignaient que cette dérivation entraîne un abaissement préjudiciable de la nappe alluviale.

Electricité de France a donc été amenée, pour étudier cette nappe alluviale, à mettre en place un réseau d'observations piézométriques qui, après quelques années d'exploitation, a permis de définir les zones où la nappe reçoit une alimentation à partir de la rivière. Ces zones sont localisées à l'aval des goulets qui délimitent 4 bassins principaux dans la basse vallée. Des démonstrations d'injection artificielle de débits comparables à ceux injectés naturellement par la rivière $\left(2 \mathrm{~m}^{3} / \mathrm{s}\right.$ au total) ont alors été entrepris dans chacune des zones.

Après des essais de principe à Plan d'Orgon (1) et à la Seignone, près d'Avignon, en 1953, l'étude systématique des écoulements non permanents en

* Ingénieur en chef à Electricité de France, Région d'Equipement Hydraulique - Alpes III.

* * Ingénieur-Conseil. Aix en-Provence - France.

(1) Exemple d'alimentation artificielle de la nappe phréatique d’un bassin alluvial de 5000 ha situé en BasseDurance», Compte rendu denxième Congrès des Irrigations et du Drainage, Vol. III, 1954 : A. Decelde, M. Guelton et R. Muller-FeUga. milieu poreux (2) a permis de mettre en place et de réaliser les démonstrations de Châteaurenard et de Peyrolles en 1956, et de Cavaillon-Cheval Blanc en 1957. L'étude de la mise en place des dispositifs et les résultats des essais sont présentés ci-après.

\section{Rappel des \\ formules d'écoulement transitoire et leur mode d'utilisation}

A partir des travaux de Boussinesq, Theis a exprimé l'évolution des niveaux d'une nappe primitivement horizontale et soumise à une injection, par la formule :

$$
h=\frac{\mathrm{Q}}{4 \pi \mathrm{KH}} \int_{0}^{u} \frac{e^{-u}}{u} d u \quad \text { avec } \quad u=\frac{r^{2} \mathrm{C}_{e}}{4 \mathrm{KH} t}
$$

où $h$ est le relèvement du niveau, $Q$ le débit injecté, K la perméabilité et $\mathrm{C}_{e}$ la capacité d'écoulement du terrain, $H$ l'épaisseur de la nappe, $r$ la distance du point de mesure au point d'injection, et $t$ le temps depuis lequel l'injection est commencée.

Meyer a étendu cette formule au cas où la nappe est primitivement en mouvement permanent, en y ajoutant un facteur correctif de la forme $e^{-(x i / 2 \mathrm{H})}$ où $x$ est l'abscisse comptée dans le sens d'écoulement de la nappe de pente $i$. Quand la pente est faible, ce qui est généralement le cas dans la nature, le terme correctif est de l'ordre de 1 à $2 \%$ et peut facilement être négligé.

Rivières, plans d'eau libre, réseaux artificiels de

(2) Celle-ci est exposée de façon plus détaillée dans l'article * Alimentation artificielle de la nappe alluviale de la Basse-Durance. Etude des dispositifs d'injection». Par M. Guelton, R. Muller-Feuga et P. Ruby, - Commission Internationale des Irrigations et du Drainage, $3^{\circ}$ Congrès. 1957, Question 8 - R. 4 


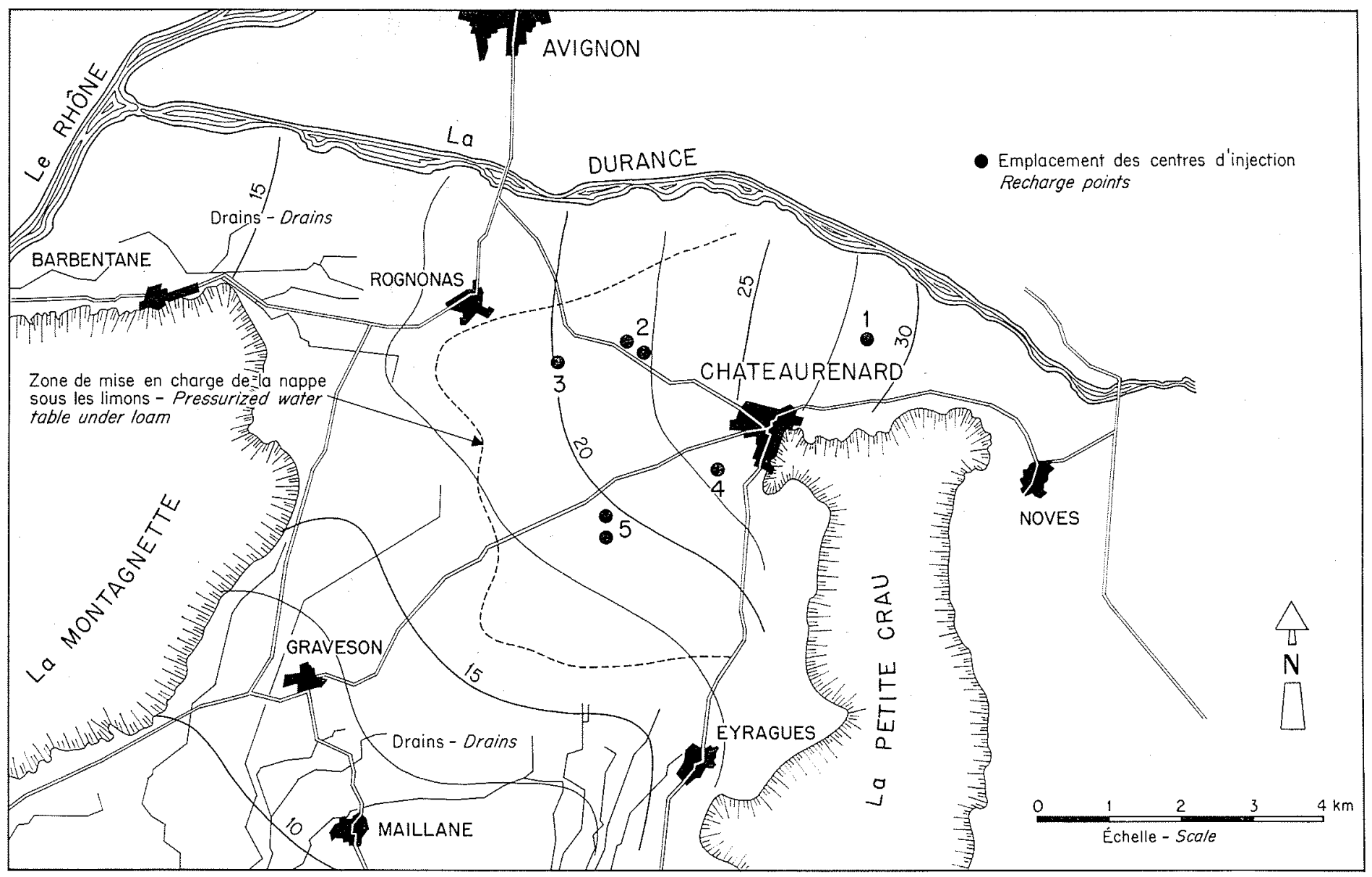

Bassin de Châteaurenard (zone de l'essai).

/1/

Châteaurenard basin (test area).

drainage, versants imperméables, etc., constituent des conditions aux limites de la nappe dont il est aisé de tenir compte par application de la théorie classique des images : un plan d'eau libre entraîne un relèvement nul et l'existence virtuelle d'un point de pompage symétrique du point d'injection par rapport à «l'écran négatif»; un versant imperméable entraine une réflexion des relèvements et l'existence virtuelle d'un point d'injection symétrique du point d'injection réel par rapport à «l'écran positif».

Enfin, les effets de plusieurs points d'injection ou de leurs images positives ou négatives dans les «écrans limites» s'ajoutent algébriquement en tous points.

\section{Données générales sur les essais}

Les 3 essais décrits dans la note devaient satisfaire à des conditions communes :

- les relèvements devaient être sensibles sans toutefois être dommageables. Ils ont généralement varié de 0,20 à $1,00 \mathrm{~m}$;

- Ies essais devaient avoir lieu en hiver (pas d'arrosage) pendant une période d'étiage de la rivière, sans pluviométrie. Ils ont eu lieu durant le mois de janvier.

Le choix de l'emplacement des points d'injection a été fait par approximations successives de façon à obtenir les relèvements désirés et à les répartir de façon homogène dans la zone visée.
Ce choix devait en outre tenir compte du fait que l'eau injectée était de l'eau brute de rivière qu'il était nécessaire de filtrer.

L'injection ne devant pas subir d'à-coups, les ouvrages de filtration devaient fonctionner de façon continue. Ils ont donc été surdimensionnés de $50 \%$, de façon que leur entretien puisse être assuré. Le filtre, en sable de diamètre effectif $d_{10}=0,42 \mathrm{~mm}$, était capable de $1 \mathrm{l} / \mathrm{s}$ par $\mathrm{m}^{2}$.

Les ouvrages d'injection étaient constitués soit de forages de $560 \mathrm{~mm}$ équipés de tubes crépinés de $300 \mathrm{~mm}$, la couronne étant remplie de graviers calibrés, soit de sondages de $114 \mathrm{~mm}$ développés par pompages saccadés par tranches de $2 \mathrm{~m}$.

\section{Alimentation artificielle de la nappe du bassin de Châteaurenard}

Le bassin alluvial de Châteaurenard est situé en rive gauche de la Durance, avant le confluent de cette rivière avec le Rhône. C'est une plaine large de 5 kilomètres environ dont la partie nord est couverte par un réseau d'irrigation très développé alors que, dans ses parties ouest et sud, un drainage systématique est pratiqué (fig. 1).

Les observations géologiques, géophysiques et hydrologiques effectuées dans la région permettent de préciser que :

- les alluvions reposent généralement sur des marnes miocènes imperméables; 
- vers le sud, elles sont recouvertes d'une couche de limons d'épaisseur souvent importante (jusqu'à 14 mètres) sous laquelle l'écoulement se fait « en charge» et qui est marquée de facon continue par des sources et par l'apparition d'un réseau de drainage évacuant les eaux de débordement de la nappe et constituant une limite de relèvement nul (écran négatif);

- les alluvions ont une épaisseur moyenne de 30 mètres et une perméabilité moyenne de $8 \cdot 10^{-3} \mathrm{~m} / \mathrm{s}$.

L'étude de la mise en place des centres a été faite en prenant pour la capacité d'écoulement $\mathrm{C}_{e}$ la fourchette 6-12\% (fig. 2).
On est finalement arrivé à 5 centres d'injection, numérotés de 1 à 5 , avec respectivement comme débits maximaux possibles : 200, 300, 100, 100 et $400 \mathrm{l} / \mathrm{s}$.

L'essai a été poursuivi pendant 29 jours, en janvier 1956 , à un débit moyen de $830 \mathrm{l} / \mathrm{s}$.

Pour faire apparaître l'effet réellement imputable à l'essai décrit, on a reconstitué, par corrélations graphiques, à partir des variations de niveaux observés sur des points d'eau comparables mais hors de portée de l'injection, ce qu'aurait été l'évolution naturelle des niveaux. On a alors tracé les courbes des relèvements à 29 jours qu'on peut comparer aux relèvements calculés pour ce délai (fig. 3).
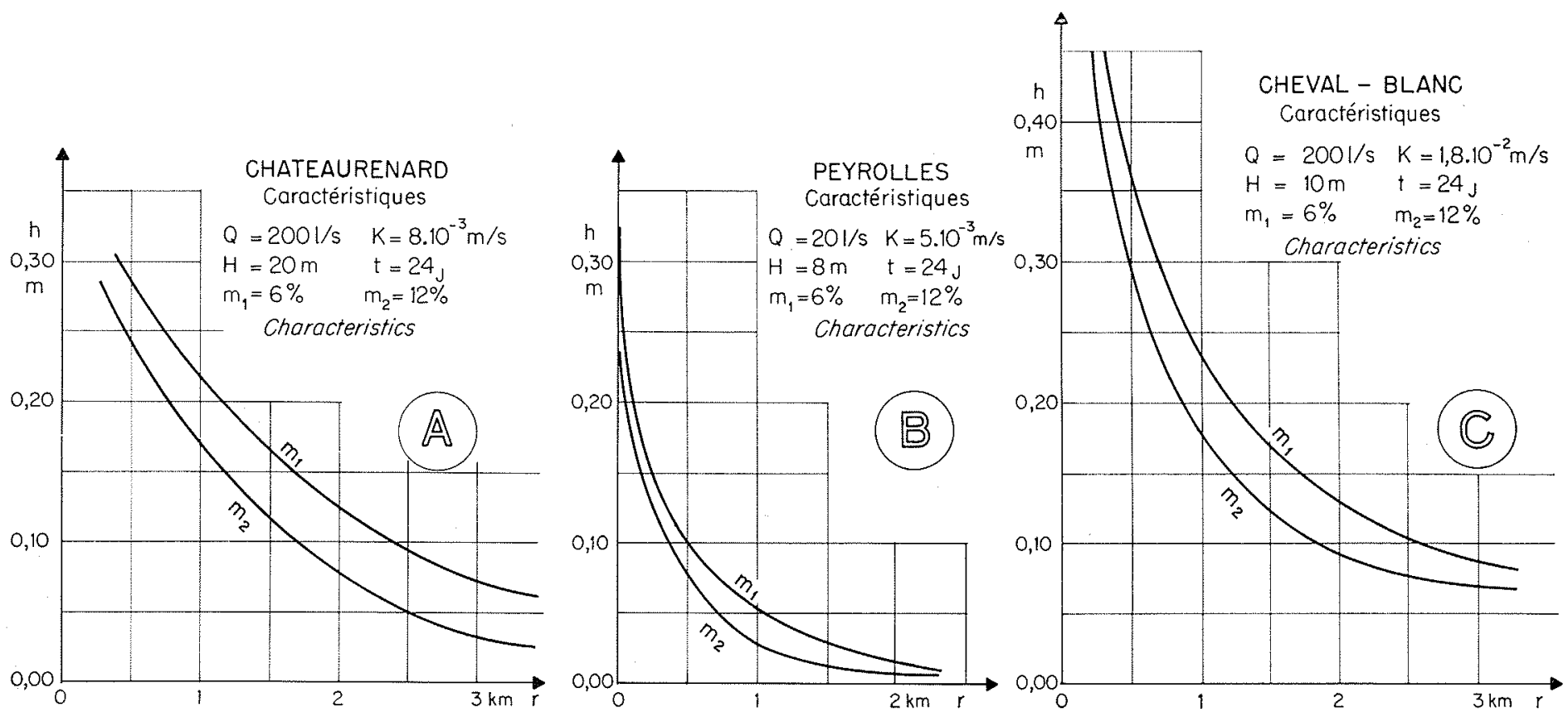

Courbes $h(r) \quad / 2 / \quad \mathrm{h}(\mathrm{r})$ curves.


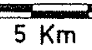

3/ Résultats de l'essai de Châteaurenard à 29 jours. Châteaurenard test results after 29 days.
Courbes de 204060.80 ef $100 \mathrm{~cm}$ de relèvement $20,40,60,80$ and $100 \mathrm{~cm}$ level rise contours

Limite de mise en charge de lo nappe sous les limons Pressurized water toble under loam 


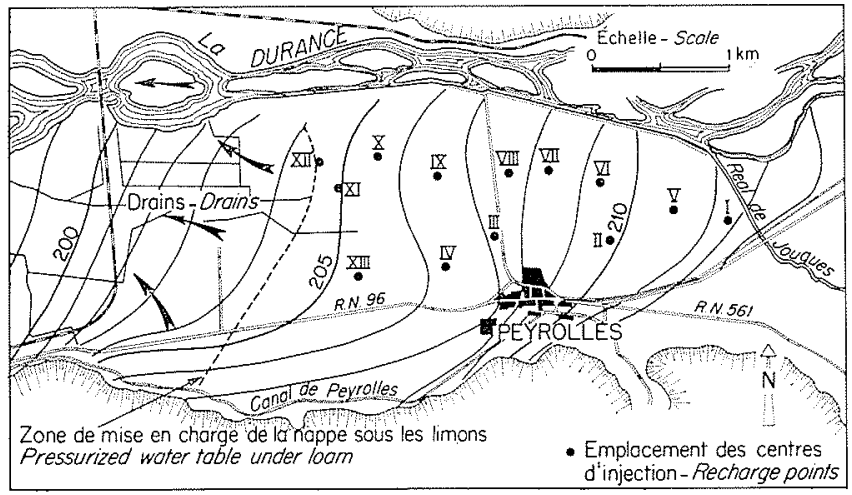

4) Zone de l'essai de Peyrolles (courbes de niveau piézométriques).

Pelyrolles test area (showing pressure levels).

\section{Alimentation artificielle de la nappe de la plaine de Peyrolles}

La plaine alluviale de Peyrolles est située en amont de la Basse-Durance et en rive gauche. Elle présente des caractéristiques générales qui la rendent très comparable au bassin de Châteaurenard et qui ont conduit à réaliser l'essai dans la partie amont de la plaine (fig. 4).

Dans cette zone, en effet, on constate que la nappe se met en charge sous une couche de limons superficiels qui est marquée de façon continue par des sources et par l'apparition d'un réseau de drainage. De plus, il existe à l'amont de la plaine une zone de hauts fonds molassiques qui marquent la limite amont de l'essai.

Les alluvions ont une épaisseur moyenne de $8 \mathrm{~m}$ environ et une perméabilité moyenne de $5 \cdot 10^{-3} \mathrm{~m} / \mathrm{s}$. Comme à Châteaurenard, on a pris pour la capacité d'écoulement la fourchette 6-12\% (fig. 2).

Cette faible épaisseur de nappe a amené la mise en place de centres d'injection plus nombreux et à débits moindres. Leur multiplication a été facilitée par la possibilité de prélever dans les rigoles de

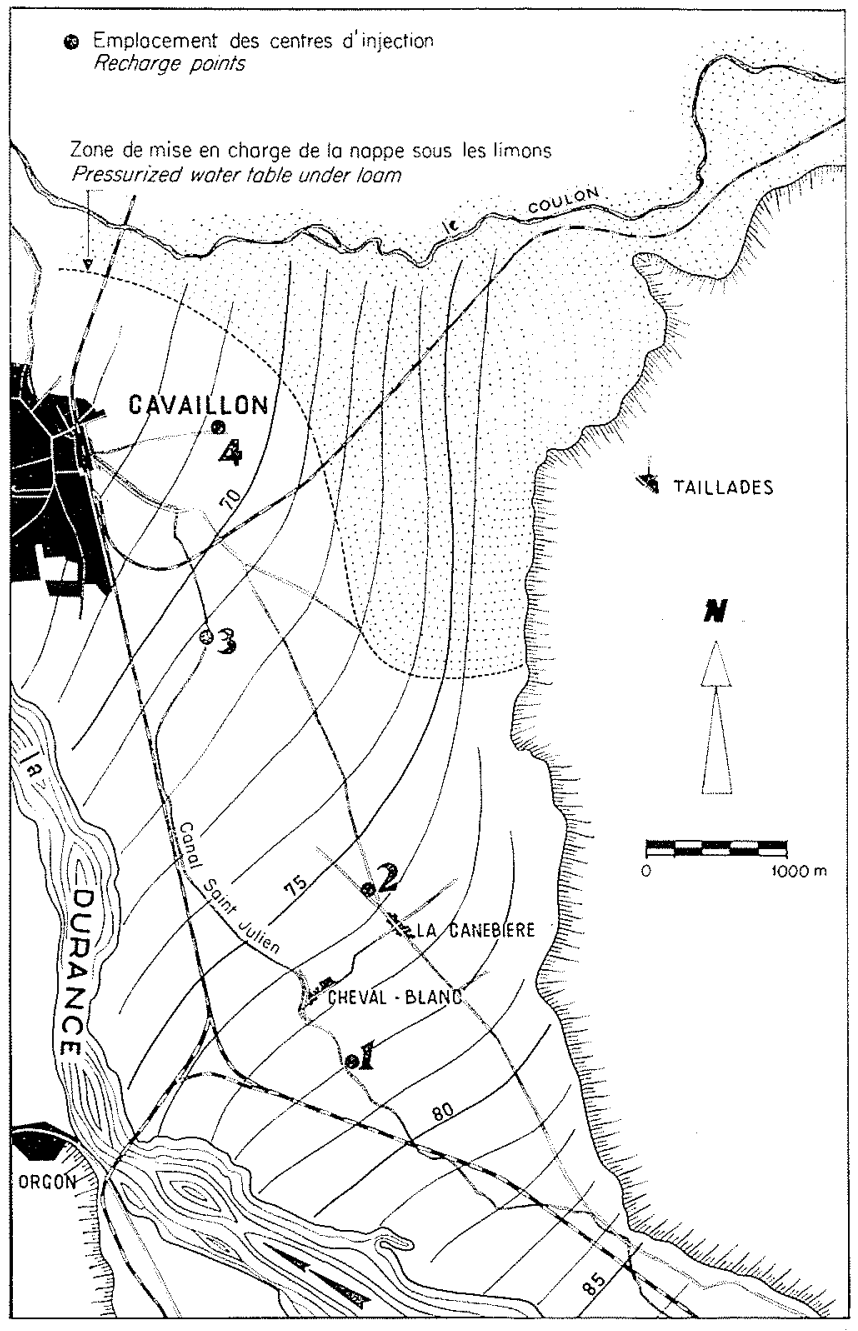

6/ Bassin de Cavaillon-Cheval Blane (zone de l'essai). Gavaillon-Cheval Blanc basin (test area).

distribution de l'eau claire fournie par des sources de versant situées à l'amont de la plaine. Il n'était pas nécessaire, de ce fait, de disposer d'ouvrages de filtration encombrants. 13 centres d'injection ont


5/ Résultats de l'essai de Peyrolles à 26 jours.

ÉCHELLE - SCALE

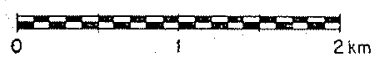

Peyrolles test results after 26 days.
Courbes de $20,40,60$ et $80 \mathrm{~cm}$ de relèvement $20,40,60$ and $80 \mathrm{~cm}$ level rise contours Zone de mișe_en chorge de la noppe sous les limons Pressurized water table under loam 


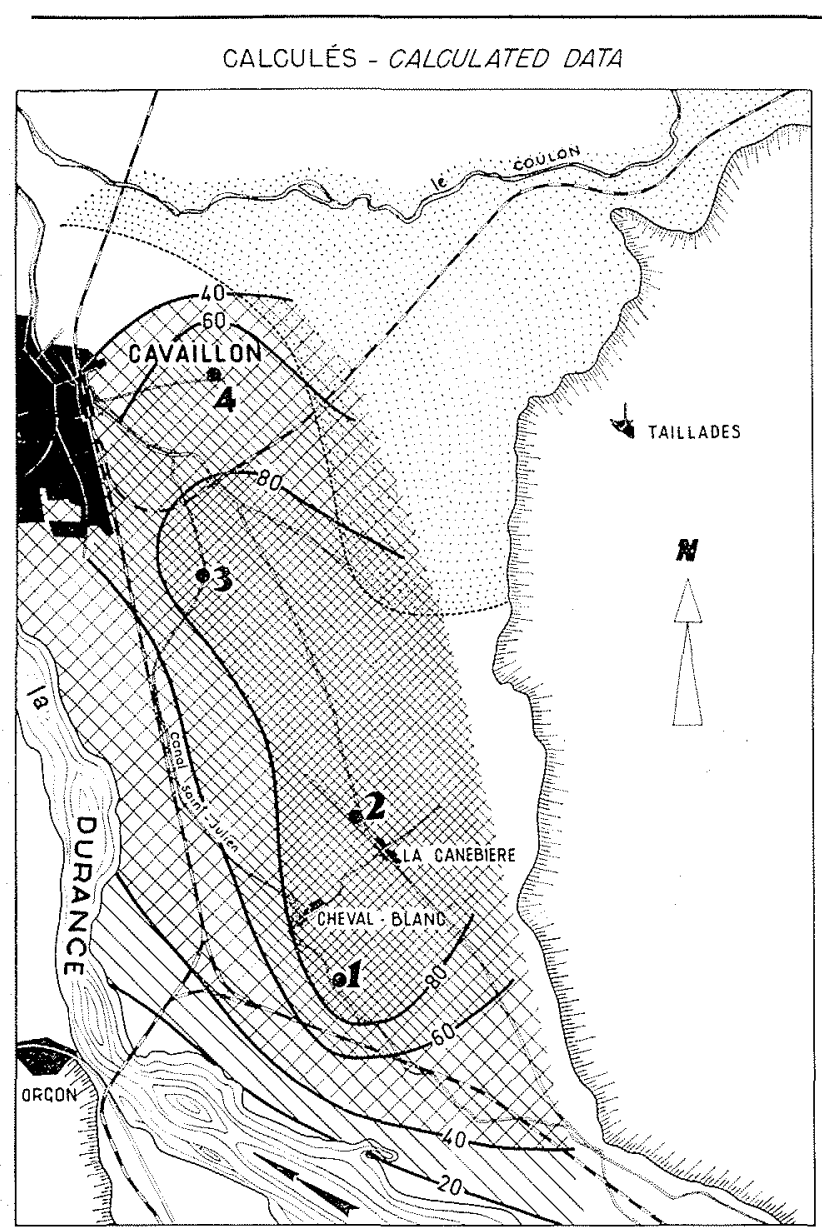

Courbes de $20,40,60,80,100 \mathrm{~cm}$ de relèvement $20,40,60,80,100 \mathrm{~cm}$ level rise contours

Zone de mise en charge de la noppe sous les limons Pressurized water fable under loam

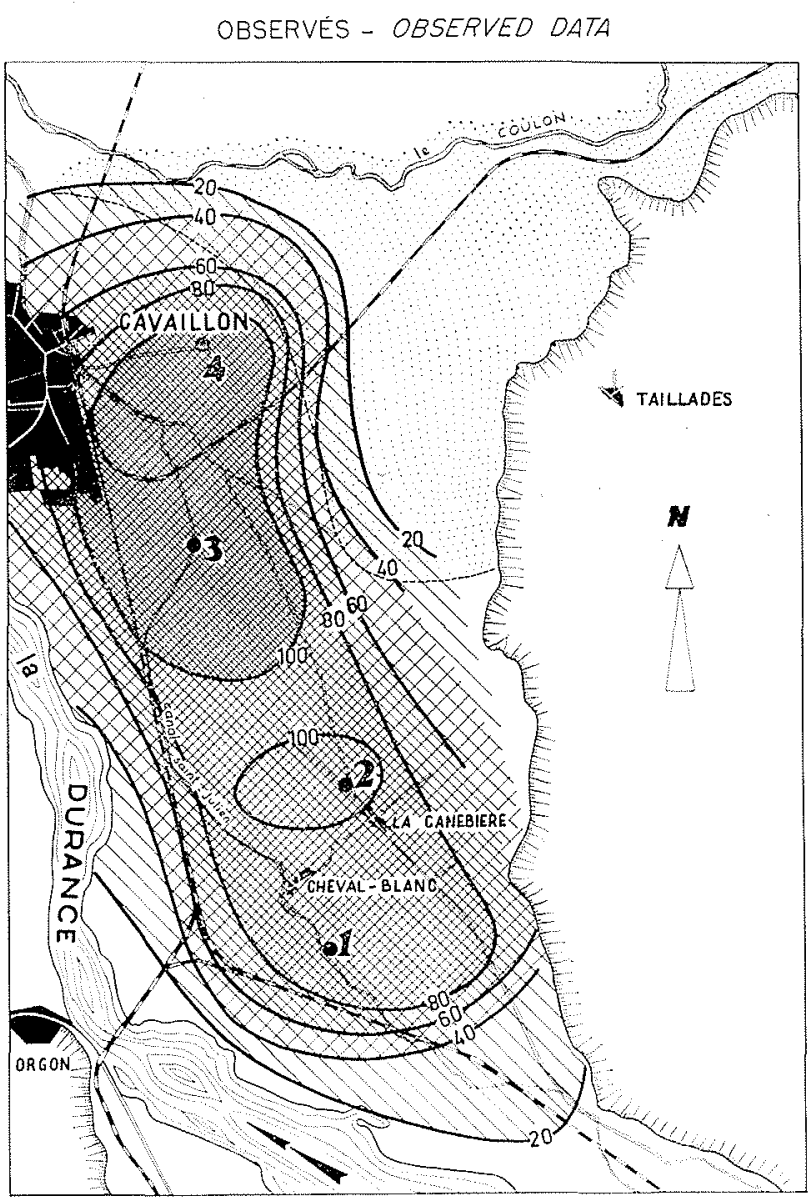

1/ Résultats de l'essai de Cheval Blanc à 29 jours. Cheval Blanc test results after 29 days. alors été mis en place, capables chacun d'un débit de $20 \mathrm{l} / \mathrm{s}$.

L'essai a été poursuivi pendant 26 jours en janvier 1956, à un débit moyen de $220 \mathrm{l} / \mathrm{s}$.

Pour faire apparaître l'effet réellement imputable à l'injection, on a reconstitué, par corrélations graphiques, à partir des variations de niveaux observées sur des points d'eau comparables mais hors de portée de l'injection, ce qu'aurait été l'évolution naturelle des niveaux. On a alors tracé les courbes de relèvements à 26 jours qu'on peut comparer aux relèvements calculés pour ce délai (fig. 5).

\section{Alimentation artificielle de la nappe du bassin de Cavaillon-Cheval Blanc}

Ce bassin se trouve en rive droite de la Durance et ses caractéristiques sont elles aussi semblables à celle du bassin de Châteaurenard : alluvions reposant sur des marnes miocènes imperméables, existence d'une couche imperméable (alluvions colmatées et limons) sous laquelle la nappe se met en charge-écoulement à surface libre, vers la Durance, ailleurs (fig. 6).

L'épaisseur des alluvions est variable de 15 à $25 \mathrm{~m}$ et leur perméabilité moyenne égale à $13.10^{-3} \mathrm{~m} / \mathrm{s}$. Cette variation de l'épaisseur de la nappe a amené à étudier l'effet de chaque centre d'injection avec des caractéristiques différentes. Mais, comme à Châteaurenard, on a pris pour la capacité d'écoulement la fourchette 6-12\% (fig. 2).

Quatre centres ont finalement été définis, numérotés de 1 à 4 , avec respectivement des débits de $100,200,200$ et $200 \mathrm{l} / \mathrm{s}$.

L'essai a été poursuivi pendant 29 jours en janvier 1957, au débit moyen de $690 \mathrm{l} / \mathrm{s}$.

Pour faire apparaitre l'effet réellement imputable à l'injection, on a reconstitué par corrélations graphiques, à partir des variations de niveaux observés sur des points d'eau comparables mais hors de portée de l'injection, ce qu'aurait été l'évolution naturelle des niveaux. On a alors tracé les courbes des relèvements à 29 jours qu'on peut comparer aux relèvements calculés pour ce délai (fig. 7).

\section{Conclusions}

La technique spéciale d'alimentation artificielle d'une nappe alluviale a été utilisée avant les essais qui viennent d'être décrits. Ces derniers constituent cependant une étape importante à cause des exigences très sévères auxquelles ils ont dû satisfaire et des résultats qui ont été obtenus. Tant sur le plan pratique que sur le plan psychologique, sans préjuger de la nécessité d'y avoir recours dans l'avenir, ils ont tranquillisé tous les intéressés, car ils ont fait apparaître que, malgré la complexité des 
questions d'écoulements souterrains qui, par cela même, sont peu connues, on disposait d'un palliatif certain en cas d'une éventuelle modification de l'écoulement de la nappe alluviale, imputable à la dérivation de la Basse-Durance.
Sur le plan général de l'hydrologie souterraine en milieu alluvial, ils ont montré que des études théoriques, généralement très ardues dans ce domaine, peuvent parvenir à une approximation très satisfaisante.

\section{Discussion}

Président : M. LEFoulox

M. le Président remercie M. Muller-Feuga de sa conférence qui a été extrêmement intéressante, puisqu'il a montrè qu'avec trois essais il avait pu convaincre les collectivités de Basse-Durance de la possibilité d'une réalimentation artificielle de la nappe. C'est d'ailleurs ce qui a valu à l'Electricité de France de pouvoir réaliser cet aménagement.

Sur la demande de M. le Président, M. Muller-Feuga indique qu'il n'a pas été nécessaire de mettre en cuvre en Basse-Durance, de facon occasionnelle ou systématique, les méthodes d'alimentation artificielle de nappe depuis que les essais décrits ont été réalisés.

Electricité de France s'attend à certains incidents sur les niveaux des nappes des parties de la vallée se trouvant à hauteur de chaque tronçon ouvert à la dérivation. Mais l'importance de ces incidents - pour autant qu'ils se produisent - a été supputée.

D’ailleurs, pour répondre à la question précise de M. le Président, les perturbations observées à hauteur de SaintEstève-Jeanson donnent la mesure de ce qui est attendu à hauteur des autres tronçons dérivés : elles n'ont pratiquement pas été ressenties par les populations locales. Tout porte à croire que l'ensemble de la dérivation de la Durance vers l'étang de Berre se soldera par des conséquences du même ordre.

Les moyens disponibles en cas de nécessité ont trouvé leur origine dans la constatation que la Durance n'intervient sur la nappe en ne l'alimentant en certains sites privilégiés et très limités que :

- en période de crues; dans ce cas, la notion d'épandage et d'accroissement sensible de la surface de percolation doit évidemment prendre le pas sur la notion de débits de la rivière. En fait, il $\mathrm{y}$ a alimentation de la nappe lorsque la rivière s'épanche largement hors de son lit mineur. - par de faibles débits; cette notion est intéressante pour l'économie du projet. Elle a été confirmée lors des 5 essais réalisés en Basse-Durance : au cours de chacun d'eux les débits mis en ouvre ont été de l'ordre de quelques centaines de litres seconde (maximum de lordre de $1 \mathrm{~m}^{3} / \mathrm{s}$ ). De tels débits ont permis, il est intéressant de le répéter, de réaliser des pointes $h(t)$ comparables à celles qui sont provoquées par de très fortes crues, et en tous cas des amplitudes de niveaux identiques à celles observées dans la nature.

\section{Discussion :}

M. Lagarde demande à M. Mulder-Feuga combien de temps a nécessité l'utilisation de la méthode qu'il a décrite (superposition des fonctions de puits par méthode graphique) et s'il ne pense pas que, pour un temps d'étude plus long que la durée citée (1 mois), sa méthode risque de devenir imprécise, par omission des différentes images qui sont à considérer dès que les limites interviennent. M. LAGARDE est d'accord avec le conférencier pour admettre que le problème essentiel à la bonne concordance des résultats de calcul et des constatations expérimentales réside dans l'adoption de valeurs de perméabilités raisonnables. Ce choix semble à M. LaGarde tellement important que l'effort principal des pétrolier's s'est porté sur ce sujet; en plus des modèles déjà cités et utilisés "itérativement " (ou par approximations successives) M. JACQUARD, chef du Département de Mathématiques Appliquées à l'Institut Français du Pétrole, a mis au point une méthode de détermination automatique, sur grand ordinateur, des perméabilités d̀ partir des essais des puits en simultané, c'est-à-dire pratiquement à partir du comportement de leurs niveaux piézométriques et quelle que soit la variation des débits dans le temps.

M. Muller-Fevga répond au premier point soulevé par M. LAGARDE :

L'étude des alimentations artificielles a connu deux phases :
Au cours de la première, on a tout d'abord confronté les caractéristiques de la nappe accessibles par la logique (zones d'alimentation, débits en cause, rôle de la rivière) ou par des mesures sur place, a celles de la documentation, quasi inexistante à l'époque, concernant les expériences de réalimentation artificielle. Les caractéristiques précitées et les possibilités de recharge artificielle ont paru devoir s'accorder et, après deux essais de principe réalisés sans calcul préalable, on a cherché à ajuster le but visé et les moyens disponibles lors d'un court essai à petit débit.

Après la phase de l'《 instruction », nous avons comnu celle des réalisations. Dans 3 bassins géographiques très diffẻrents de la vallée, nous avons réalisé un essai. L'étude propre à chaque essai a duré 4 à 5 jours: en gros, le projeteur réalisait en une journée, sur les données déjagées par un ingènieur, des courbes $h(r)$ (à 3 temps différents) avec une répartition de débits donnée par centre. An bout de ce délai, après des ajustements de répartition de débits et d'implantations des centres, on avait trouvé des formes de courbes de relèvement satisfaisantes à 31 jours.

Pour répondre au deuxième point soulevé par M. LAGarde, on doit rappeler que nous nous étions proposé pour objectif, au cours des essais, de passer en un mois des niveaux minimaux annuels connus par la nappe aux niveaux maximaux moyens annuels rencontrés généralement en juilletaoût. Il n'aurait pas été possible de poursuivre les essais au-delà du délai d'un mois sans dépasser les niveaux rencontrés naturellement et, par conséquent, sans risquer de provoquer des préjudices pour lesquels nous n'aurions pas manqué d'être recherchés.

Si, ayant atteint le but visé en superficie et en amplitudes, nous avions été conduits à prolonger dans le temps l'alimentation artificielle, il nous aurait fallu soit réduire les débits injectés (il faudrait voir dans ce cas si la répartition des centres demeurerait convenable), soit procéder par pulsations (pour éviter l'incidence précitée). On n'aurait pas eu de nouvelles images à mettre en cause et la méthode aurait conservé sa précision.

Au cas particulier, il est intéressant de préciser que si les essais ont montré qu'on ponvait relever des niveaux de façon sensible, en fait, l'objectif pour l'avenir se limiterait pour E.D.F. à maintenir des niveaux, ce qui conduirait à mettre en œuvre des débits plus faibles que ceux des essais.

M. Ruby indique, au sujet de la comparaison des problèmes pétroliers, qu'il ne faut pas perdre de vue la différence qui existe entre les conditions de gisement du pétrole et celles de l'eau. Outre que les pétroliers peuvent mobiliser des moyens d'étude importants, l'échelle à laquelle ils travaillent est généralement très différente de celle que connaissent les hydrauliciens, et les méthodes de travail des premiers ne sont pas forcément applicables aux seconds.

Notamment, les hydrauliciens travaillent sur des valeurs des paramètres hydrodynamiques bien supérieures à celles qu'on connaît dans le pétrole, et dans ces conditions, l'influence des limites ne se pose pas avec la mème acuité.

Les relèvements de niveaux piézométriques obtenus lor's de l'essai de Châteaurenard, par exemple, où le coefficient de stockage de la nappe est de l'ordre de $10 \%$, sont d'environ $1,50 \mathrm{~m}$ autour des centres d'injection pour une épaisseur initiale de nappe de $30 \mathrm{~m}$. Dans ces conditions, l'influence des «limites» du bassin (lesquelles se trouvaient à des distances de l'ordre de 1 à $2 \mathrm{lsm}$ des centres d'injection), si elle est sensible, n'est quand même pas très importante, et il a suff de tenir compte des images des puits d'injection au premier degré seulement. Sauf exception, on n'a pas cu à tenir compte des images d'imases.

Le problème de la superposition des relèvements à divers temps est done simple et se résout graphiquement sans difficulté, ce qui explique qu'il n’ait pas été nécessaire de faire appel, dans de pareils cas, aux modèles analogiques. 
M. Tixeront demande à M. Muller-Feuga de parler de son système d'injections.

M. Muller-Fruga mentionne que les injections ont été réalisées, après filtrations, par des sondages. Le système de filtration utilisé était le même pour tous les centres : chacun d'eux comporte 3 bassins capables de filtrer ensemble $150 \%$ du débit pour lequel le centre est prévu; deux de ces bassins fonctionnent en permanence pendant que le troisième est en réserve ou a son filtre en cours de rénovation. Les superficies des filtres ont été calculées à raison de $1 \mathrm{n}^{2}$ par litre à la seconde de débit à filtrer. En coupe verticale, on rencontre une conche de sable de $0,30 \mathrm{~m}$ reposant sur des dalles de béton poreux d'environ $0,20 \mathrm{~m}$ : les eaux filtrées sont distribuées après mesure des débits vers les points d'injection. On mettait le filtre d'un bassin en rénovation lorsque la charge sur celui-ci atteignait $0,80 \mathrm{~m}$ environ. Sauf en périodes de crues, durant lesquelles les eaux étaient sales puisque les eaux alimentant les centres provenaient de la Duranee par le réseau d'irrigation très faiblement utilisé en hiver, deux hommes pouvaient assurer l'entretien de 2 centres d'alimentation artificielle. Il est à noter qu'en période d'hiver, les crues sont très rares.

Le système d'injections était constitué par des forages. On a exécuté des forages de $1 \mathrm{~m}$, de $0,56 \mathrm{~m}$ et de $0,10 \mathrm{~m}$ de diamètre. Dans ce dernier cas, le forage était particulièrement développé en provoquant une aspiration la plus vive possible sur la plus petite tranche possible de crépine; on procédait en remontant. Dans la pratique, les sondages de $0,10 \mathrm{~m}$ se sont comportés de façon quasi identique aux forages de $0,56 \mathrm{~m}$.

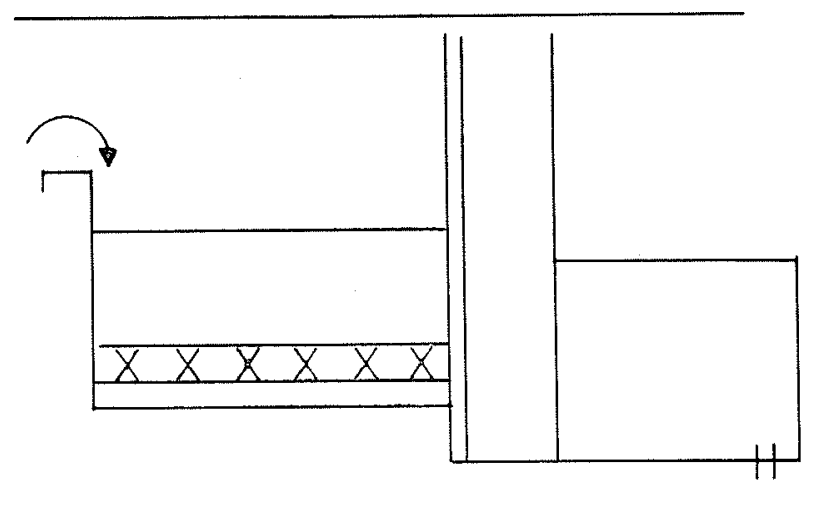

Après le premier essai, pour lequel des forages de $1 \mathrm{~m}$ de diamètre ont été réalisés, les résultats nous ont conduits à n'utiliser que des sondages de $0,10 \mathrm{~m}$. Nous n'avons pas observé de tendance au colmatage systématique. Par contre, dès les tout premiers essais, il nous est apparu qu'il y avait intérêt à éviter la formation de vortex ou de toute turbulence à l'entonnement dans les forages, car on observait le blocage progressif de l'absorption et par conséquent un abaissement du coefficient de Darcy du terrain par dégagement de l'air contenu dans l'eau. 